ARTICLE

\title{
Fast widefield scan provides tunable and uniform illumination optimizing super-resolution microscopy on large fields
}

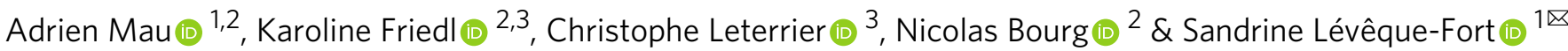

Non-uniform illumination limits quantitative analyses of fluorescence imaging techniques. In particular, single molecule localization microscopy (SMLM) relies on high irradiances, but conventional Gaussian-shaped laser illumination restricts the usable field of view to around $40 \mu \mathrm{m} \times 40 \mu \mathrm{m}$. We present Adaptable Scanning for Tunable Excitation Regions (ASTER), a versatile illumination technique that generates uniform and adaptable illumination. ASTER is also highly compatible with optical sectioning techniques such as total internal reflection fluorescence (TIRF). For SMLM, ASTER delivers homogeneous blinking kinetics at reasonable laser power over fields-of-view up to $200 \mu \mathrm{m} \times 200 \mu \mathrm{m}$. We demonstrate that ASTER improves clustering analysis and nanoscopic size measurements by imaging nanorulers, microtubules and clathrin-coated pits in COS-7 cells, and $\beta 2$-spectrin in neurons. ASTER's sharp and quantitative illumination paves the way for high-throughput quantification of biological structures and processes in classical and super-resolution fluorescence microscopies.

\footnotetext{
${ }^{1}$ Institut des Sciences Moléculaires d'Orsay, Université Paris-Saclay, CNRS, Orsay, France. ${ }^{2}$ Abbelight, Cachan, France. ${ }^{3}$ Aix-Marseille Université, CNRS, INP UMR7051, NeuroCyto, Marseille, France. ${ }^{\circledR}$ email: sandrine.leveque-fort@universite-paris-saclay.fr
} 
$\mathrm{n}$ advanced widefield fluorescence microscopy, lasers are a common excitation source: they provide excitation at precise wavelengths coupled with high control over the delivered power, both properties that are crucial to obtain quantifiable images. Typically, the laser is focused at the back focal plane (BFP) of the objective to produce a large, collimated beam illuminating the whole sample. While widefield fluorescence microscopy is a fast imaging method, resulting images are usually contaminated by blur from below and above the plane of focus, clouding the fluorescence signal.

Optical sectioning improves the signal by spatially limiting the illumination around the focal plane: highly inclined and laminated optical sheet (HiLo) excitation translates the laser beam in the BFP to an oblique illumination of the sample ${ }^{1}$. Placing the beam at the position corresponding to illumination at the critical angle results in a "grazing incidence", $\sim 1-\mu \mathrm{m}$ thick illumination sheet above the coverslip ${ }^{2,3}$. Inclining the beam further ${ }^{4}$ results in total internal reflection fluorescence (TIRF) ${ }^{5}$, restraining the illumination to an exponentially decreasing intensity over a few tens of nanometers above the coverslip surface. These remarkable sectioning capabilities can be performed on one single setup $^{6-8}$ and allow to study membrane and adhesion processes with minimal background. In practice, however, TIRF suffers from heterogeneous illumination caused by the interference patterns arising from the high spatial coherence of lasers and scattering. Rapidly spinning the beam around the BFP can alleviate these fringes by averaging beam orientations over a single camera frame 9 , a method since applied with several variants and refinements ${ }^{10-12}$.

The methods above result in Gaussian-shaped illumination profiles over the sample. This is sufficient for the typical field of view (FOV) acquired by EMCCD cameras but is more problematic over larger FOVs acquired by newer, highly sensitive sCMOS cameras ${ }^{13}$. The non-uniformity of Gaussian-shaped illumination lowers exploitable FOV sizes and thereby decreases the imaging throughput, a significant caveat for quantitative analysis of images obtained by TIRF.

The need for uniform excitation is even more pressing in single-molecule localization microscopy (SMLM) such as (f) PALM $^{14,15}$ or PAINT ${ }^{16}$ where the localization precision strongly varies with the number of emitted photons. It is even more considerable in (d)STORM ${ }^{17-19}$, where the single-molecule regime $\left(\ll 1\right.$ emitting molecule $\left./ \mu \mathrm{m}^{3}\right)$ relies on driving most fluorophores in a dark state, provided through a high irradiance $\left(\mathrm{kW} / \mathrm{cm}^{2}\right)$. As the transition to the dark state is highly dependent on the local excitation intensity, non-uniformities of illumination result in a strongly heterogeneous blinking behavior and loss of image quality across the FOV. For all SMLM methods, no matter the origin of the single-molecule emission, non-uniform precision precludes proper analysis of SMLM images over large fields of view.

Thus, several recent studies have aimed at obtaining a uniform excitation over a large FOV. For example, waveguides ${ }^{20-22}$ provide excellent fixed TIRF on large fields, but cannot be restricted to the actual FOV acquired by the camera, illuminating and bleaching the whole sample at once. Classical solutions revolve around beam-reshapers ${ }^{23-26}$ and multimode fibers ${ }^{27-29}$ but are also restrained in field adaptability. Spatial light modulators $\left(\right.$ piSMLM ${ }^{30}$ ) may adapt the shape and size of the FOV but suffer from high-power loss and are rather expensive and complex. All these classical methods illuminate the whole field at once, so they may be ill-adapted to TIRF (see Supplementary Note 1), need speckle reducers and provide larger FOVs under the premise of using higher laser power. Additionally, focusing a high-power laser beam at the edge of the BFP may damage the lens at the back of the objective.
To circumvent the compromise between laser power requirements, optical sectioning performances, and field uniformity, we developed Adaptable Scanning for Tunable Excitation Region (ASTER). ASTER is a hybrid scanning and widefield excitation scheme that can perform epifluorescence, oblique, or TIRF illumination, while providing illumination uniformity at variable FOV sizes adapted to the camera or sample. Being a general widefield illumination scheme, ASTER can benefit to both classical widefield fluorescence microscopy and SMLM.

\section{Results}

Flat-top epifluorescence/TIRF excitation principle. ASTER is a hybrid scanning and widefield excitation scheme. Any classical widefield setup can be converted into ASTER configuration by smoothly integrating alternative optical conjugation along with a scanning device such as galvanometers. In our implementation (Fig. 1a), the initial Gaussian beam, which provides a limited and non-uniform excitation, is focalized between two galvanometer scanning mirrors placed in a plane conjugated to the BFP of the objective so that an angle shift applied to the mirrors will induce a similar angle shift in the objective BFP and a position shift of the beam at sample plane. This configuration allows for large $X-Y$ area scans of a collimated beam. Fast scanning of the Gaussian beam position in defined patterns such as raster scan or an Archimedes spiral then generates an overall homogeneous illumination (Supplementary Fig. 1) over the FOV when averaged over the camera frame exposure time. The field size can be increased or diminished in milliseconds without physical intervention by adapting the galvanometer input amplitude. Notably, as the polar angle of the beam varies at the BFP while its position is maintained, this flat-top excitation scheme is compatible with inclined illumination such as oblique or TIRF. To this end, a conventional motorized translation stage serves as switch from epifluorescence to oblique and TIRF excitation.

To generate a uniform flat-top excitation over the whole FOV, the scanning needs to meet two criteria. First, the maximum distance between adjacent lines on the beam path has to be lower than $1.7 \sigma$ (Supplementary Fig. 1), $\sigma$ being the standard deviation of the input Gaussian excitation beam. Interestingly, decreasing that gap will not affect the flat-top so that smaller gaps may be used. For a given field size, this spatial rule defines the minimum number of lines needed to achieve homogeneity (Supplementary Note 2). Second, to avoid stroboscopic effects the flat-top must be synthetized under a scanning period Tscan that divides the camera integration time Tint (Fig. 1d). A typical galvanometer mirror has a repositioning delay of $300 \mu \mathrm{s}$, so the number of scanned lines will set the minimal required time to synthesize the flat-top profile. Our implementation uses an input excitation beam of $\sigma=17 \mu \mathrm{m}$ and gaps between 1.2 and $1.4 \sigma$ : Ten lines are sufficient to generate a flat-top profile on a $200 \mu \mathrm{m} \times 200 \mu \mathrm{m}$ FOV under $5 \mathrm{~ms}$, which is two times the maximum frame rate of classical sCMOS (100 fps) cameras. In practice, we used camera integration times between 50 and $100 \mathrm{~ms}$ and a scanning period of half the integration time so that the flat-top was averaged twice over a single frame, though this number may be modified by adapting the scanning period, so that the flat-top is averaged either once, or multiple times (Supplementary Fig. 2). Notably, compared to confocal laser scanning ASTER does not perform point scanning but a continuous scan with a wide input collimated beam and thus can cover large areas much faster.

To characterize the illumination homogeneity and validate our simulations, we imaged a thin layer of fluorescent Nile Blue (Fig. 1b, c) with a classical wide Gaussian beam excitation $(\sigma=$ $45 \mu \mathrm{m})$ and with our ASTER illumination scanning a raster pattern of $150 \mu \mathrm{m}$ long lines $(\sigma=17 \mu \mathrm{m})$. Figure $1 \mathrm{c}$ shows that the 
a

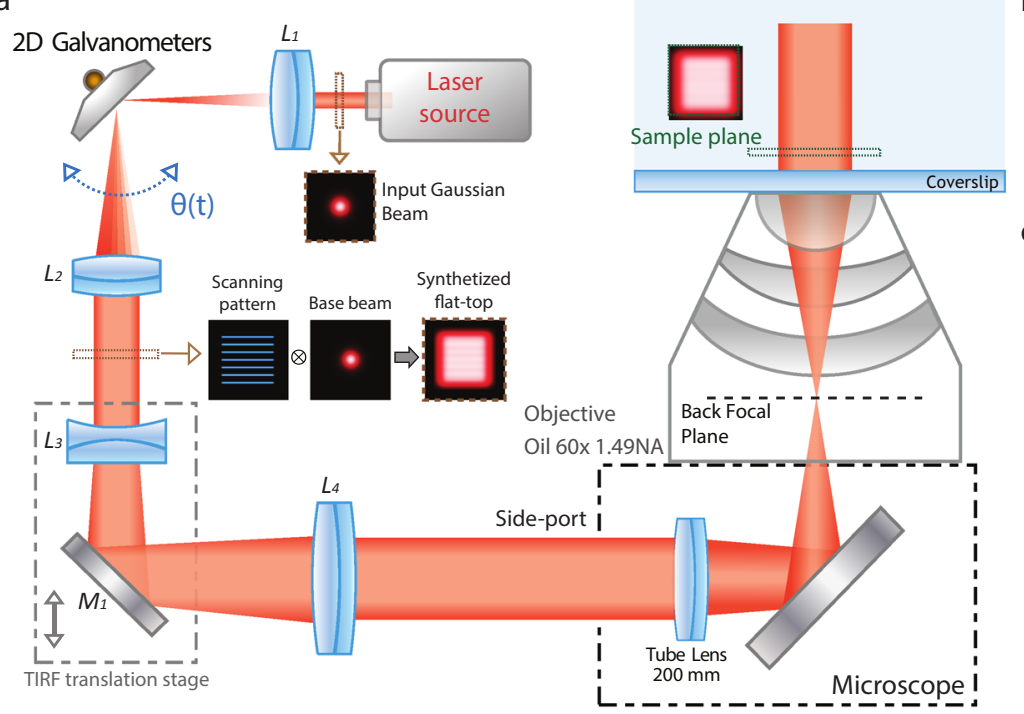

$b$
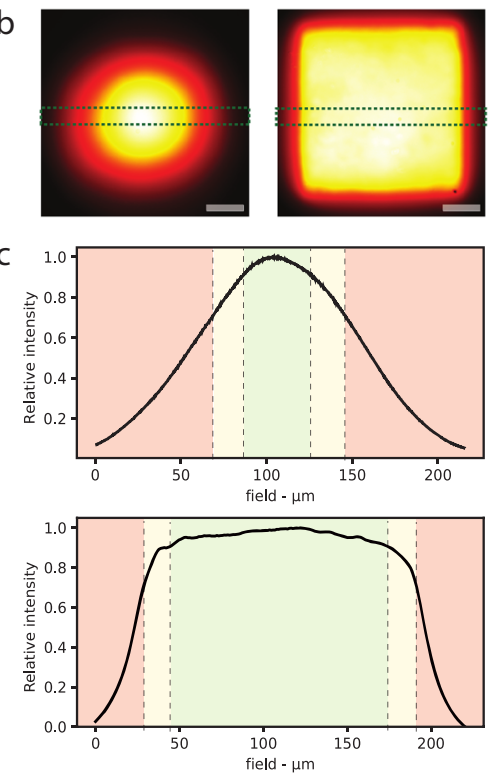

d

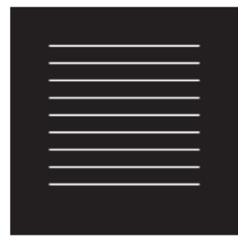

scanning path

Pattern integration over time

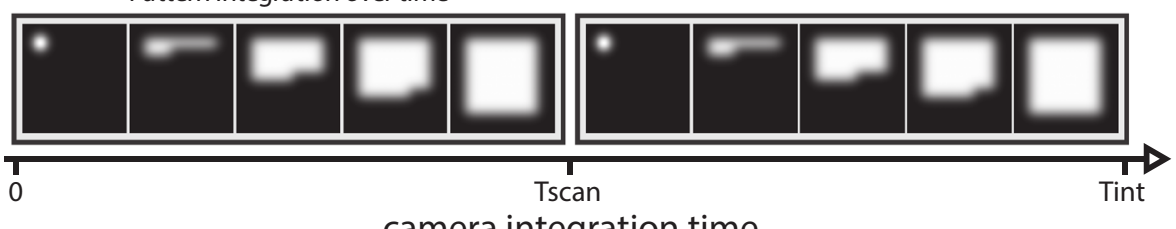

camera integration time

Fig. 1 Schematic of ASTER and resulting illumination patterns. a Simplified schematic of ASTER setup generating a homogeneous field using a raster scanning pattern. $L_{i}$ are lenses with focal length fi: $f 1=100, f 2=100, f 3=-35, f 4=250 . M_{1}$ is a dielectric mirror. A small input Gaussian base beam is scanned in-between the $L_{1}$ and $L_{2}$ lenses, resulting in a collimated flat-top profile, which then goes through a TIRF translation stage and is magnified between $L_{3}$ and $L_{4}$. After focalization at the BFP of an objective lens, it results in a temporally averaged flat-top excitation profile at the sample. $\mathbf{b}$ Thin layer of fluorescent Nile Blue imaged at low laser power with a fixed Gaussian excitation beam (left) and with ASTER (right) raster scanning excitation. Scalebars $40 \mu \mathrm{m}$. $\mathbf{c}$ intensity profiles from $\mathbf{b}$ of Gaussian (up) and ASTER (bottom) illuminations taken along the green dashed area. Dashed lines and colors indicate the field ranges in which intensity is above $90 \%$ (green), between 70 and $90 \%$ (yellow) or below $70 \%$ (red) of its maximum value. d Scanning path (left) and generation of the uniform profile over temporal acquisition of the camera. (right). With Tint the camera integration time, the example of a scanning period $\operatorname{Tscan}=$ Tint $/ 2$ is shown.

ASTER illumination triggers homogeneous Nile Blue fluorescence over a single camera frame, with a square shape matched to typical camera detectors. The resulting flat-top illumination profile is consistent with our simulations and exhibits significant flatness over $\sim 130 \mu \mathrm{m}$, which could be diminished or increased by adapting the galvanometer input amplitude. In this configuration, if we consider that intensity should remain over $90 \%$ of its maximal value for confident quantification over the FOV, the Gaussian illumination would be limited to a $32 \mu \mathrm{m} \times 32 \mu \mathrm{m}$ usable FOV, while ASTER can provide at least a $\sim 16 \mathrm{X}$ larger, $130 \mu \mathrm{m} \times$ $130 \mu \mathrm{m}$ FOV. On Fig. 1c ASTER exhibits Gaussian-shaped borders that reflects the use of a base Gaussian beam of $\sigma=17$ $\mu \mathrm{m}$. A smaller base Gaussian beam may be used to sharpen the flat-top borders, but at the cost of slower imaging speed as more lines will have to be scanned. The decrease in brightness at the periphery of the image also stems from vignetting, an effect occurring on all microscope objectives ${ }^{31}$ as light beams emanating from the periphery of the field are partially blocked by optical or mechanical components. We confirm this phenomenon by scanning a large flat-top illuminating the full field of the camera (Supplementary Fig. 3). With our 60X magnification and square fields, vignetting is negligible for fields smaller than $160 \mu \mathrm{m} \times 160$ $\mu \mathrm{m}$, at $200 \mu \mathrm{m} \times 200 \mu \mathrm{m}$ up to $21 \%$ intensity is lost at the corners (affecting 9\% of the field), this increases up to $35 \%$ loss of intensity on the full $220 \mu \mathrm{m} \times 220 \mu \mathrm{m}$ field of our camera (affecting $18 \%$ of the field). In conclusion, even though a wide uniform excitation can be provided, homogeneity is ultimately limited by detection to uniform fields of $160 \mu \mathrm{m} \times 160 \mu \mathrm{m}$, and relatively uniform fields of $200 \mu \mathrm{m} \times 200 \mu \mathrm{m}$. By working on a circular field, however, a vignetting-free area of $200 \mu \mathrm{m} \times 200 \mu \mathrm{m}$ (radius of $113 \mu \mathrm{m}$ ) can be specifically illuminated by scanning an Archimedes spiral (Supplementary Fig. 1).

We then assessed the compatibility of ASTER with inclined, optically sectioning illumination schemes, where a precise alignment and focusing of the excitation beam in the BFP of the objective is crucial (Supplementary Fig. 4). We focused on TIRF, as it is one of the most common schemes used in SMLM. First, we compared TIRF to classical epifluorescence illumination (EPI) obtained through ASTER by imaging 3- $\mu \mathrm{m}$ diameter beads, coated with biotin, and labeled with AF647-streptavidin. As can be assessed on Supplementary Fig. 5, due to the spherical shape of the beads the experimental sectioning depth is reflected through the beads apparent size ${ }^{32,33}$ : imaging of beads from EPI to TIRF resulted in the shrinking of the beads radii. This effective sectioning stems from both optical sectioning of the illumination and depth of field of our objective. We then imaged beads on a $160 \mu \mathrm{m} \times 160 \mu \mathrm{m}$ FOV and measured their respective size in EPI and TIRF (Fig. 2a, b). To assess the existence of spatial correlation, we measured the sectioning depth of each individual sphere that we define as twice the height of the intensity ring. The 

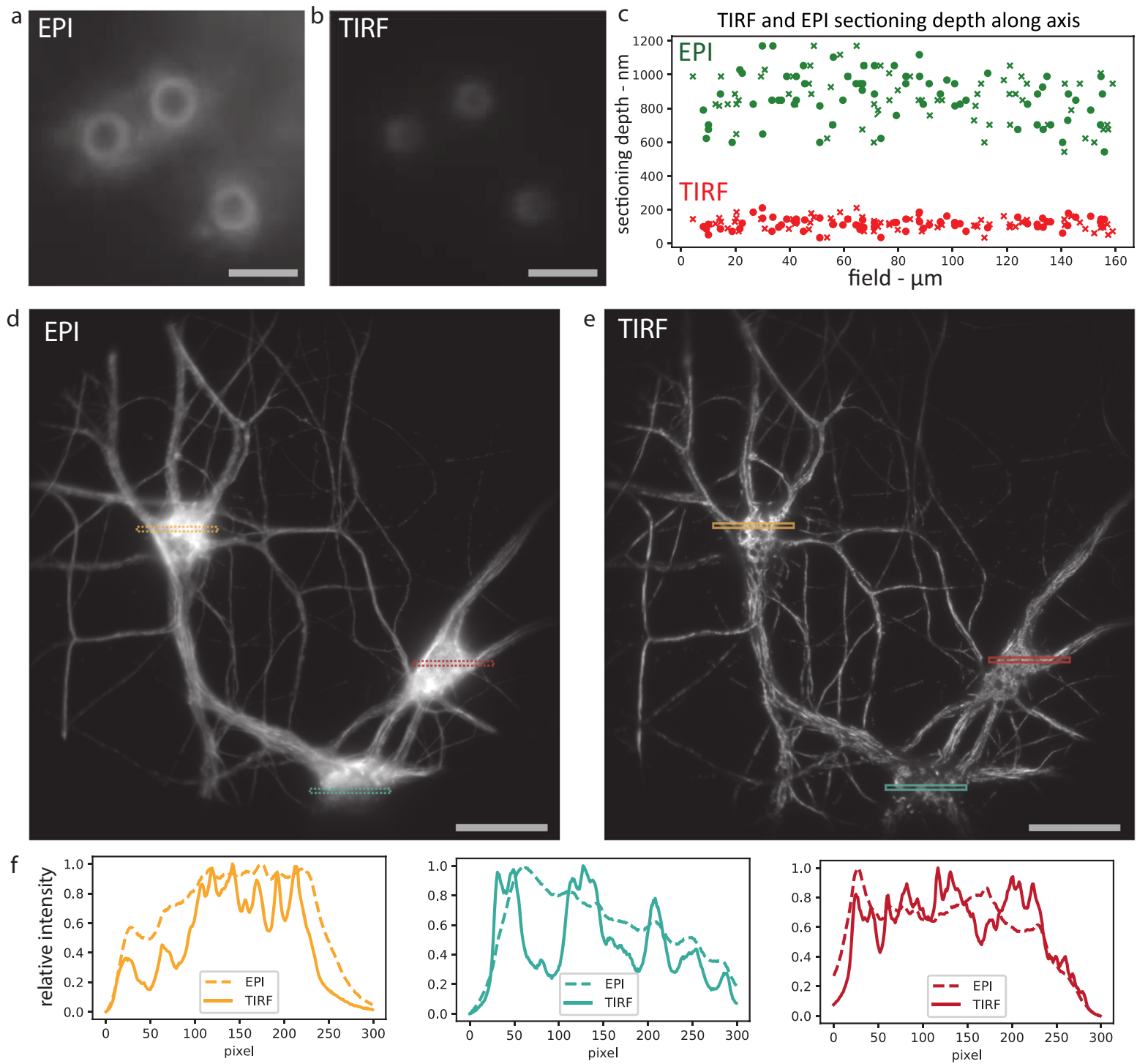

Fig. 2 ASTER TIRF illumination. Illumination of $3 \mu \mathrm{m}$ beads with focus at the coverslip, in ASTER epifluorescence (EPI, a) and ASTER TIRF (b) illuminations (whole images are shown in Supplementary Fig. 6). Scalebars $4 \mu \mathrm{m}$. c Measured sectioning depth for 67 individual beads in EPI (green) and TIRF (red) illuminations on a large $160 \mu \mathrm{m} \times 160 \mu \mathrm{m}$ FOV. Cross and circle markers, respectively, denote measurement along the $x$ and $y$ axis of the sample plane. In total, $200 \mu \mathrm{m} \times 200 \mu \mathrm{m}$ imaging FOV of neurons labeled with an anti- $\beta 2$-spectrin primary and an AF647-coupled secondary antibody, illuminated with raster scanning ASTER, with a scanning period of $50 \mathrm{~ms}$ and an exposure time of $100 \mathrm{~ms}$, in either classical epifluorescence (d) or TIRF (e) illumination schemes. Scalebars $40 \mu \mathrm{m}$. $\mathbf{f}$ Normalized EPI and TIRF profiles of each colored area in $\mathbf{d}$ and $\mathbf{e}$.

measured sectioning depth for TIRF excitation is $117 \pm 35 \mathrm{~nm}$ (mean \pm standard deviation), while epifluorescence yields a depth of $865 \pm 149 \mathrm{~nm}$, likely defined by the objective's depth of field (Fig. 2c). The sectioning depths for both schemes are uniform over the FOV, and their variations show no local or global spatial correlation (Supplementary Fig. 6 and Supplementary Note 3), demonstrating the absence of a spatial excitation anisotropy. Variation between beads most likely stem from both measurement precision and physical discrepancy of the bead population.

To assess the optical sectioning efficiency in biological samples, we imaged rat hippocampal neurons labeled for 32 -spectrin, a submembrane scaffold protein lining the neuronal plasma membrane, revealed with AF647. We compared EPI and TIRF configurations on a $200 \mu \mathrm{m} \times 200 \mu \mathrm{m}$ FOV (Fig. 2d, e). The images show that ASTER with TIRF maintains the quality of optical sectioning along the whole FOV: fluorescence over the cell bodies of neurons (parts that are thicker than the sectioning depth) exhibit less blurred fluorescence and a better signal can be observed compared to the epi-illuminated image, revealing the delicate structure of the neuronal network (Fig. 2f). While a $200 \mathrm{~ms}$ integration time was used to improve signal to noise ratio, ASTER can provide uniform TIRF excitation under $5 \mathrm{~ms}$ integration times (Supplementary Fig. 7), which makes it adapted to imaging fast live dynamical processes. A disadvantage of TIRF with classic Gaussian-shaped laser beams are interference patterns: TIRF with ASTER, by contrast, exhibits no such inhomogeneous patterns (Supplementary Fig. 8), as they are likely to be averaged out by beam scanning and camera integration. Even though ASTER is still subject to shadowing effects, it solves both the issues of TIRF interference fringes and nonuniform Gaussian illumination, with the benefit of a large achievable FOV. In conclusion, ASTER leads to TIRF images with a similar quality as spinning azimuthal TIRF, with the added benefit of field uniformity and FOV size versatility. ASTER illumination efficiently provides both uniform spatial illumination and uniform axial optical sectioning for fluorescence microscopy in both EPI and TIRF illumination. 


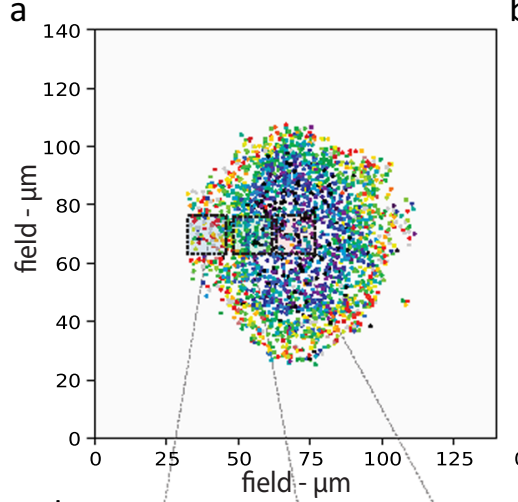

d

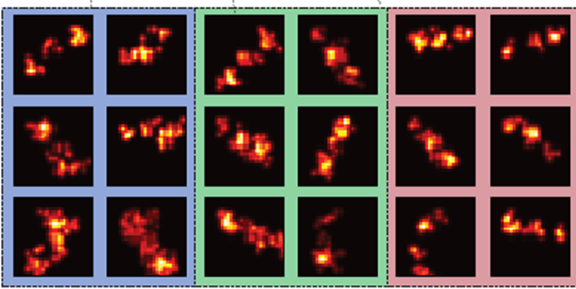

g

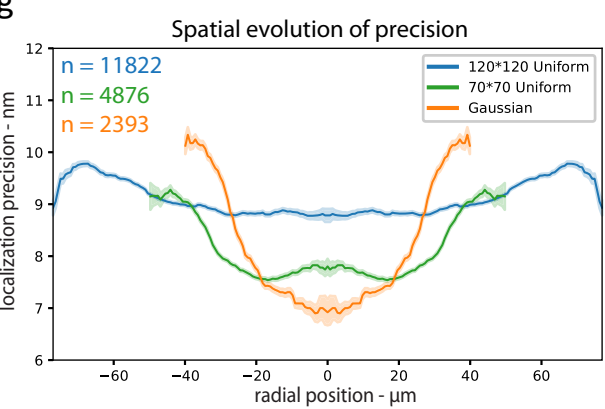

$\mathrm{h}$ b

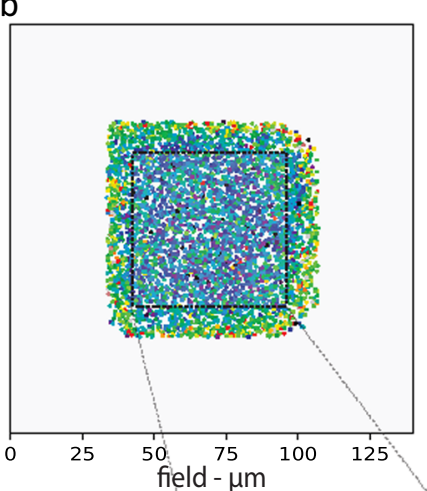

e
C

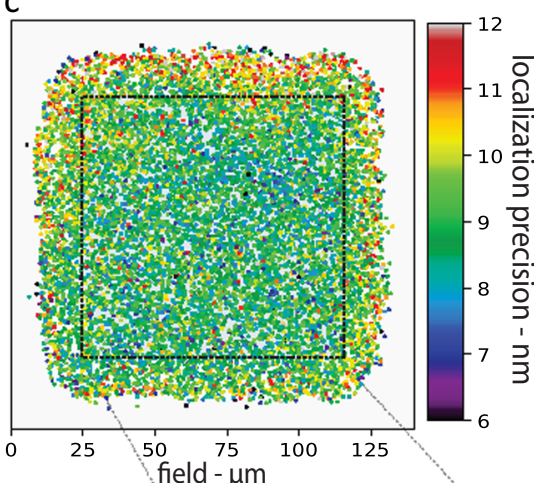

$f$
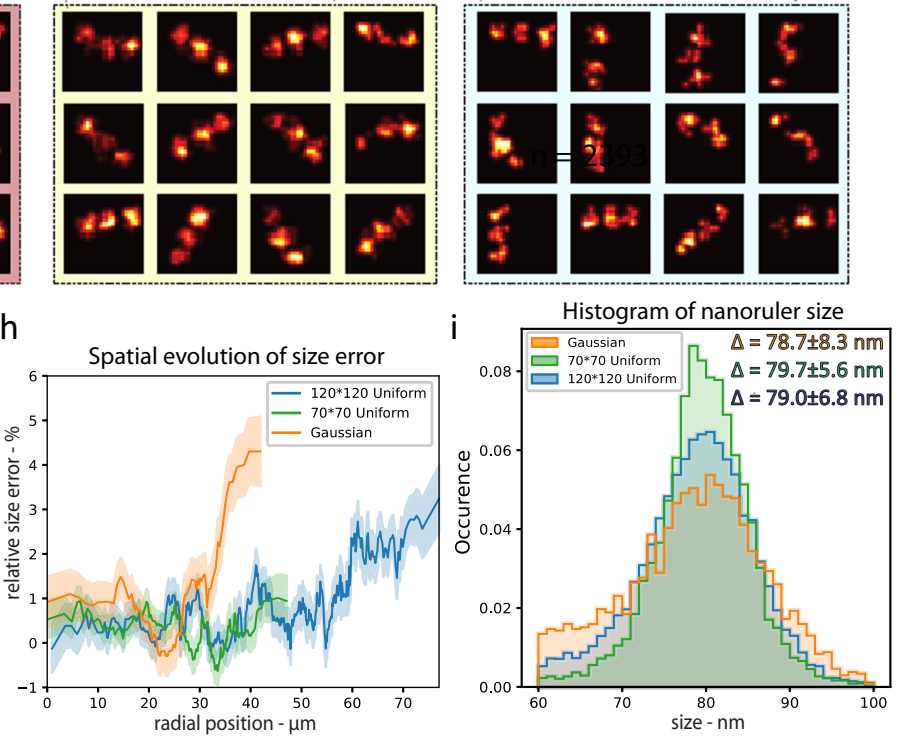

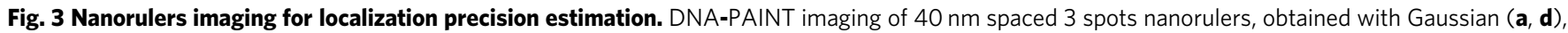
ASTER small field of view $(70 \mu \mathrm{m} \times 70 \mu \mathrm{m}, \mathbf{b}, \mathbf{e})$, and ASTER large field of view $(120 \mu \mathrm{m} \times 120 \mu \mathrm{m}, \mathbf{c}, \mathbf{f})$ illuminations. a-c are resulting localization precision maps where each point represents the average precision for one individual nanoruler ( 3 spots). d-f are nanoruler super-resolution images ( 150 nm $\times$ $150 \mathrm{~nm}$ ), taken randomly from highlighted areas in a-c. $\mathbf{g}$ Mean localization precision along FOV radius for each excitation scheme (symmetrized). The number of nanoruler for each excitation scheme is indicated in a similar color. For each colored curve, the surrounding transparent curve indicates the standard deviation around the mean precision at a given radius. $\mathbf{h}$ Resulting size estimation error along FOV radius for each excitation scheme. A size error above 0 indicates that the nanoruler spots were measured $<80 \mathrm{~nm}$ apart. Each colored curve is surrounded by a transparent curve that indicates the standard deviation around the mean size. i Resulting size measurement histogram for each excitation scheme. The mean and the standard deviation for the size (symbolized by $\Delta$ ) are indicated in the upper right corner.

Large field uniform SMLM imaging. Next, we applied ASTER to SMLM experiments, namely DNA-Point Accumulation in Nanoscale Topography (DNA-PAINT) and STochastic Optical Reconstruction Microscopy (STORM). To assess the effect of ASTER illumination FOV size and homogeneity in SMLM experiments, and compare it to a classical Gaussian illumination, we first imaged three spots, $40 \mathrm{~nm}$ spaced nanorulers using DNAPAINT (Fig. 3). Three different TIRF excitation schemes (Gaussian, $\sigma=45 \mu \mathrm{m}$ ), ASTER on a $70 \mu \mathrm{m} \times 70 \mu \mathrm{m}$ FOV, and ASTER on $120 \mu \mathrm{m} \times 120 \mu \mathrm{m}$ FOV were used, with the other parameters remaining identical. In the single-molecule regime, each of the three nanoruler spot acts as a source of blinking fluorescence, resulting in a set of localizations spread by the pointing accuracy of each blinking event. For analysis we applied the following algorithm: first, individual nanorulers were isolated by DBscan clustering ${ }^{34}$, then for each individual nanoruler the point cloud corresponding to the three spots was fitted by a Gaussian mixture model (GMM) assuming three normal distributions. The GMM assessed the most probable mean position and standard deviation of each spot (see "Methods", Supplementary Fig. 9). For each individual nanoruler, the mean standard deviation of all spots was then considered as a local measure of the experimental localization precision.

The Gaussian excitation resulted in a bell-shaped localization precision map (Fig. 3a, g): at the center of the FOV, the localization precision is $7 \mathrm{~nm}$, but it quickly increases with the distance from the center. At $20 \mu \mathrm{m}$, it is $8 \mathrm{~nm}$, and up to $11 \mathrm{~nm}$ at the edges of the FOV, 1.6 times worse than at the center (Fig. 3d, g). The mean localization precision is then $8.7 \pm 1.8 \mathrm{~nm}$ (mean \pm standard deviation). Meanwhile, ASTER excitation on a similar FOV provided a localization precision of $7.9 \mathrm{~nm} \pm 0.9 \mathrm{~nm}$-ranging from 7.5 to $8 \mathrm{~nm}$ at $30 \mu \mathrm{m}$ from the center of the field (Fig. $3 \mathrm{~b}, \mathrm{~g}$ ). On a large $120 \mu \mathrm{m} \times 120 \mu \mathrm{m}$ FOV with similar parameters, ASTER provided a $9.2 \mathrm{~nm} \pm 1.1 \mathrm{~nm}$ localization precision (Fig. 3c, g), from 8.8 at the center of the field up to $9.5 \mathrm{~nm}$ at a $60 \mu \mathrm{m}$ radial distance. This means that a $20 \mathrm{X}$ increase in the FOV size came at the cost of a 1.2 worse localization precision. It is conceivable that a localization precision below $9 \mathrm{~nm}$ could be reached by carefully optimizing imaging parameters such as laser power, optical sectioning, and camera integration time. Moreover, the inhomogeneity of the 

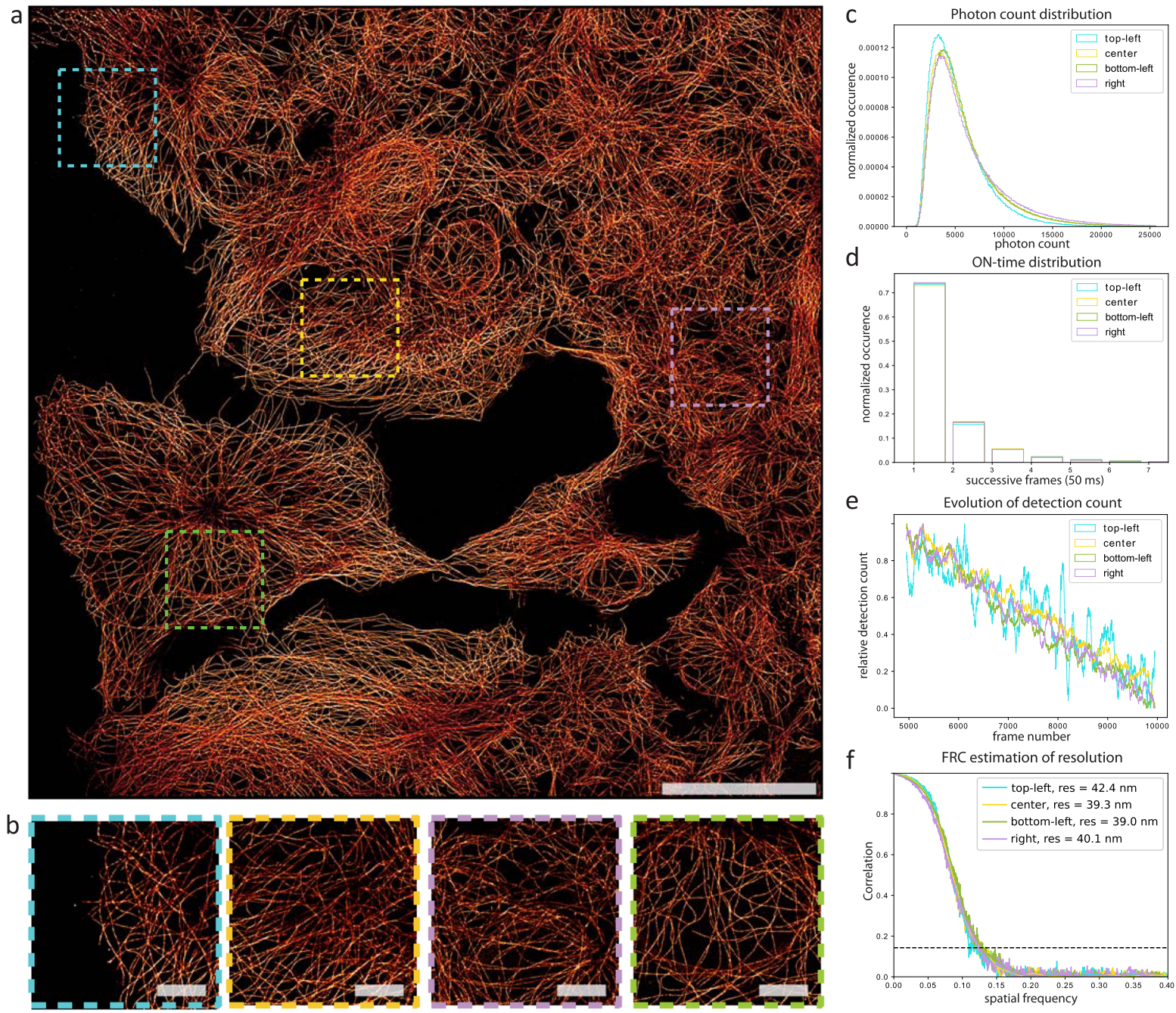

Fig. 4 STORM imaging using ASTER. a ASTER STORM imaging of COS-7 cells labeled for microtubules and an AF647-coupled secondary antibody, FOV size $200 \mu \mathrm{m} \times 200 \mu \mathrm{m}, 20,000$ frames at $20 \mathrm{fps}$. Excitation consisted in a ten-line scan with a laser power of $250 \mathrm{~mW}$ at the BFP, a gap of $1.4 \sigma$ and a $25 \mathrm{~ms}$ scanning period. Scalebar $50 \mu \mathrm{m}$. b Zoomed views of highlighted areas in $\mathbf{a}$. Scalebars $10 \mu \mathrm{m}$. c Photon count distribution histogram for highlighted areas in a. d Blinking ON-time distribution for highlighted areas in a, expressed in number of successive frames (50 ms camera integration time). e Temporal evolution of detection count for highlighted areas in a. f FRC estimation of resolution for highlighted areas in a.

Gaussian illumination impacted the size estimation of the nanorulers along the FOV (Fig. 3h). We measured the end-toend size of the identified nanorulers (ground truth value $80 \mathrm{~nm}$ ). Gaussian beam illumination images yield a fairly constant relative size error of $1 \%$ at the center of FOV, rising to $3 \%$ at $35 \mu \mathrm{m}$ from the center. ASTER provided homogeneous measurements on a wider FOV: the relative size error remained at $0.5 \%$ up to $45 \mu \mathrm{m}$ from the center of the FOV. All the illumination conditions resulted in similar mean values for the nanoruler size (Fig. 3i), but we observed an increased number of cases where the size was underestimated to $60-70 \mathrm{~nm}$ with the Gaussian beam illumination, indicating a poor single-molecule regime.

We then turned to STORM experiments on biological samples. Traditionally, STORM demands strong laser power $\left(>2 \mathrm{~kW} / \mathrm{cm}^{2}\right)$ to drive organic fluorophores into a blinking regime ${ }^{35,36}$. To induce a satisfactory blinking regime on a $200 \mu \mathrm{m} \times 200 \mu \mathrm{m}$ FOV thus requires the use of $1-5 \mathrm{~W}$ power lasers ${ }^{27}$.

However, as ASTER provides locally high excitation irradiance on a short time scale, and a lower global average excitation on longer scales, it may partly overcome this irradiance threshold rule. We applied ASTER in HiLo to a STORM experiment and found that even with reasonable laser power $(<0.3 \mathrm{~W}$ at BFP),
ASTER was able to induce and maintain a densely labeled sample in the sparse single-molecule regime $\left(<1\right.$ molecule per $\left.\mu \mathrm{m}^{3}\right)$ on large FOVs (Fig. 4), where conventional illumination would fail. It appears that the high but intermittent local excitation intensity $\left(\sim 12 \mathrm{~kW} / \mathrm{cm}^{2}\right)$ nonetheless sends most of the molecules in a longlived dark state efficiently, as is expected for high irradiances ${ }^{37}$.

Figure $4 \mathrm{a}$ shows a SMLM image of a COS-7 cell labeled for microtubules and an AF647-coupled secondary Fab 2 antibody. It was obtained using 20,000 frames and a $50 \mathrm{~ms}$ camera integration time, using ASTER with $0.3 \mathrm{~W}$ laser power, raster scanning a 200 $\mu \mathrm{m} \times 200 \mu \mathrm{m}$ FOV (ten lines). The microtubules are well resolved throughout the whole FOV; the zoomed images in Fig. $4 \mathrm{~b}$ show the image quality in several different parts of the image. Analysis of these regions revealed comparable photon distributions, blinking ON-time and localization density during acquisition (Fig. 4c-e). Even though a slight decrease in photon count can be noticed at the edge, other blinking characteristics remain unchanged and suggest once again inhomogeneous detection due to vignetting at the periphery of the objective. Analysis from regions of an image acquired with a classical Gaussian illumination showed significant differences between the regions, underlining the detrimental effect of inhomogeneous Gaussian 
illumination (Supplementary Fig. 10a-f). We assessed the experimental image resolution with Fourier ring correlation analysis $^{38}$ (Fig. 4f), and found that the region subject to vignetting had a close resolution $(42 \mathrm{~nm})$ to the other areas $(39-40 \mathrm{~nm})$ indicating that vignetting does not significantly impact the uniformity on our FOV. This confirms ASTER's ability to obtain uniform blinking and resolution on large $200 \mu \mathrm{m} \times 200 \mu \mathrm{m}$ FOVs in STORM. Noteworthy, we do not notice artifact emerging from the temporal scanning of the beam, indicating that the position of a fluorophore relative to the scanning part does not matter. Further experiments (Supplementary Fig. 11) suggest that STORM blinking properties remain similar as long as the same mean irradiance is provided.

ASTER thus is compatible with both DNA-PAINT and STORM experiments even with typical lasers currently used on SMLM microscopes with output power below $1 \mathrm{~W}$. In SMLM, because of a required pixel imaging size around $100 \mathrm{~nm}$, camera chip finite size will ultimately limit the FOV, the largest uniform FOV reported so far being $221 \mu \mathrm{m} \times 221 \mu \mathrm{m}$ by Zhao et al. ${ }^{27}$. However, their implementation did not perform TIRF and required multiple lasers with $>1 \mathrm{~W}$ output power plus a vibration motor to reduce speckles. Generally, imperfections from the detection path will limit the maximum achievable FOV. To overcome this limit, we stitched four $160 \mu \mathrm{m} \times 160 \mu \mathrm{m}$ uniform STORM images, resulting in a $300 \mu \mathrm{m} \times 300 \mu \mathrm{m}$ image (Supplementary Fig. 12a, b) with minimal overlap and high uniformity. Stitching results in minimal artifacts in the overlapping edge areas, but slightly suffers from temporal effects on photon count and molecule density, mostly due to buffer consumption between acquisitions (Supplementary Fig. 12c). To limit temporal effects, one may choose to speed up STORM experiments by increasing the global irradiance ${ }^{37,39}$. With ASTER, this can be done by reducing the amplitude of scanning. We scanned five $25-\mu \mathrm{m}$ long lines in $5 \mathrm{~ms}$ to reach an effective irradiance of $27 \mathrm{~kW} / \mathrm{cm}^{2}$, this allowed to perform fast STORM imaging of microtubule in under $100 \mathrm{~s}$ on a classical FOV (Supplementary Fig. 13). Such experiments are less prone to drift and highlight the practical versatility of ASTER for optimizing STORM experimental needs ${ }^{40}$.

As ASTER homogenously illuminates large FOVs, it extends the possibility of quantitative analysis of nanoscopic structures to whole cells or group of cells. To obtain a precise view of a biological structure at the nanoscale, it is crucial to leverage the imaging of a large number of similar structures. This allows to not only obtain their average characteristics, but also the individual variation of these characteristics caused by biological variability. We imaged clathrin clusters and clathrin-coated pits by STORM in COS-7 cells (Fig. 5a-c) and applied a cluster analysis. Three COS-7 cells were imaged at once over a large $140 \mu \mathrm{m} \times 140 \mu \mathrm{m}$ FOV, containing $\sim 20,000$ individual clathrin clusters. In comparison, a classical $30 \mu \mathrm{m} \times 30 \mu \mathrm{m}$ FOV would have yielded $\sim 1500$ pits. The high number of clathrin clusters identified on resolution-uniform images allowed for population estimation from the characteristics of clusters. We picked specific parameters such as diameter and hollowness (see "Methods"). We were able to distinguish four populations from the cluster diameter distribution, as fitted with normal distributions (Fig. 5b). Smalldiameter clusters (below $80 \mathrm{~nm}$, blue and green population of Fig. 5b) likely correspond to pits in formation, while large ones (orange and red populations in Fig. 5b) are likely to be fully assembled pits. We specifically extracted large, hollow clathrin assemblies based on the ratio between the diameter and the spatial dispersion of fluorophores. Hollow clathrin assemblies with diameters of $80-200 \mathrm{~nm}$ would be of typical size for the large clathrin-coated pits found in fibroblasts ${ }^{41}$. Interestingly, some large, hollow pits showed more than one fluorescence "holes" within them, suggesting that they are either assemblies of smaller pits or that the fenestration of clathrin cages $^{42}$ (pentagon or hexagons of $18 \mathrm{~nm}$ side length) can sometimes be resolved (Fig. 5c).

The large FOV provided by ASTER illumination coupled with large-chip sCMOS cameras also have interesting application for imaging neuronal cells, which grow axons over hundreds of microns in culture. Traditionally, SMLM imaging of axons has been limited to $<50 \mu \mathrm{m}$ segments of axons, impeding the visualization of rare structures and the definition of their largescale organization ${ }^{43,44}$. We labeled rat hippocampal neurons for $\beta 2$-spectrin, a protein that forms a periodic submembrane scaffold along axons by linking actin rings ${ }^{45-47}$. A $200 \mu \mathrm{m} \times$ $200 \mu \mathrm{m}$ FOV allowed visualizing the dendrites and cell body of two neurons, and a large number of long axonal segments (Fig. $5 \mathrm{~d}$ and Supplementary Fig. 14). The zoomed views confirm the quality and resolution of the resulting image: the periodic $190 \mathrm{~nm}$ organization of axonal spectrin is clearly visible, as confirmed by the corresponding Fourier transform of the images. The Fourier transform of the whole image exhibits a sharp ring at the corresponding frequency, because the banded pattern of $\beta 2$ spectrins appears in axons running in all directions. On the zoomed images (Fig. 5e), the $\beta 2$-spectrin along axons in one direction results in a direction-dependent frequency band on the Fourier transform, corresponding to the $190 \mathrm{~nm}$ spacing.

\section{Discussion}

We implemented and characterized ASTER, a hybrid scanning and widefield illumination technique for optimized widefield fluorescence microscopy and SMLM over large FOV. ASTER generates uniform excitation over a tunable FOV without limiting acquisition speed. It has advantages over state-of-the-art uniform illumination schemes by its efficiency, flexibility, and ability to perform uniform optical sectioning schemes, such as HiLo and TIRF illuminations. We demonstrate TIRF imaging on rat hippocampal neurons on $200 \mu \mathrm{m} \times 200 \mu \mathrm{m}$, the maximal uniform FOV achievable with our $\times 60$ magnification objective. With DNA-PAINT, we demonstrate a uniform localization precision over large FOVs $(9.2 \pm 1.1 \mathrm{~nm}$ over $120 \mu \mathrm{m} \times 120 \mu \mathrm{m})$, and even better localization precision on small FOVs $(7.9 \pm 0.9 \mathrm{~nm}$ over 70 $\mu \mathrm{m} \times 70 \mu \mathrm{m})$.

ASTER also proved to be an efficient excitation method for STORM imaging experiments. Against common belief that STORM requires a strong continuous irradiance $\left(\sim 2 \mathrm{~kW} / \mathrm{cm}^{2}\right)$, ASTER induced uniform blinking dynamics at lower mean irradiance $\left(<0.5 \mathrm{~kW} / \mathrm{cm}^{2}\right)$, but with a high instantaneous irradiance $\left(\sim 12 \mathrm{~kW} / \mathrm{cm}^{2}\right)$ over a large $200 \mu \mathrm{m} \times 200 \mu \mathrm{m}$ FOV, alleviating the need for expensive and dangerous high-power lasers. We present biological applications by directly imaging the periodic $190 \mathrm{~nm}$ organization of axonal spectrin on several long axon segments from neurons. By imaging clathrin-coated pits in multiple COS-7 cells in one acquisition, we increase the number of identified clusters by a factor of 20 compared to the typical FOV of a STORM acquisition and enhance statistical analysis.

ASTER can be combined with stitching schemes, alternative objectives, adaptive detection setups, and camera chips to cover even wider FOV. Combination of ASTER with the ASOM ${ }^{48,49}$ could be particularly interesting as hyper large fields may be imaged without moving the sample. In SMLM, the field is regularly limited to a maximum of $200 \mu \mathrm{m} \times 200 \mu \mathrm{m}$, however in classical widefield microscopy ASTER can be used with smaller magnification objectives to image larger FOVs and would be a great choice for imaging structures on larger scales. We conclude that ASTER represents a versatile and innovative tool, especially suited for SMLM. It exhibits robust uniformity and reliability, as well as adaptability to variable FOV sizes. ASTER 
a
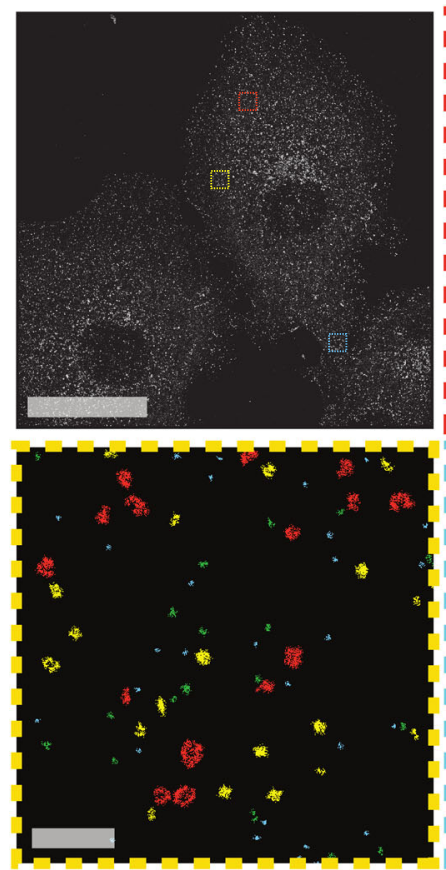

d

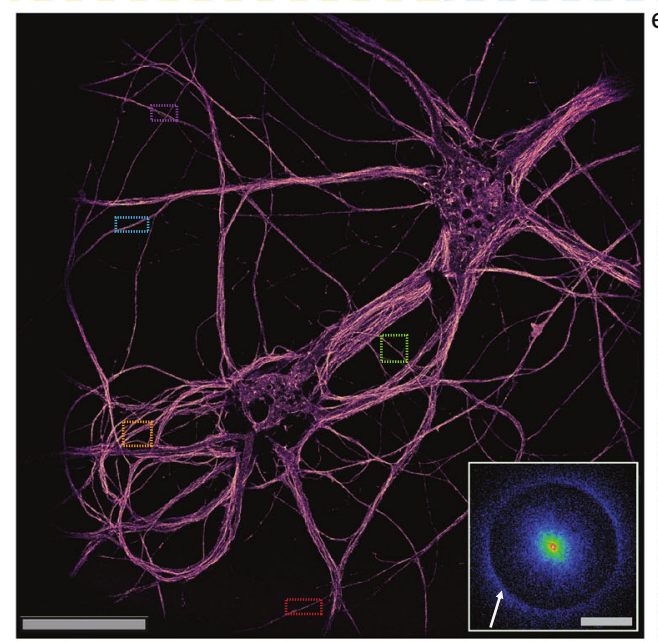

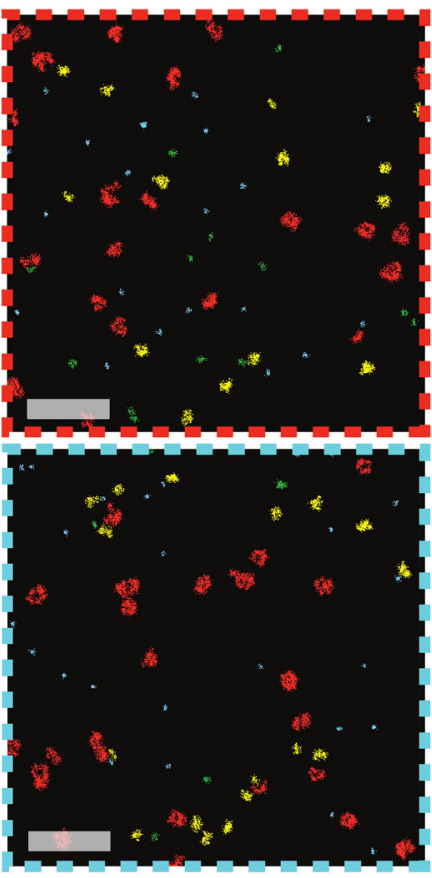
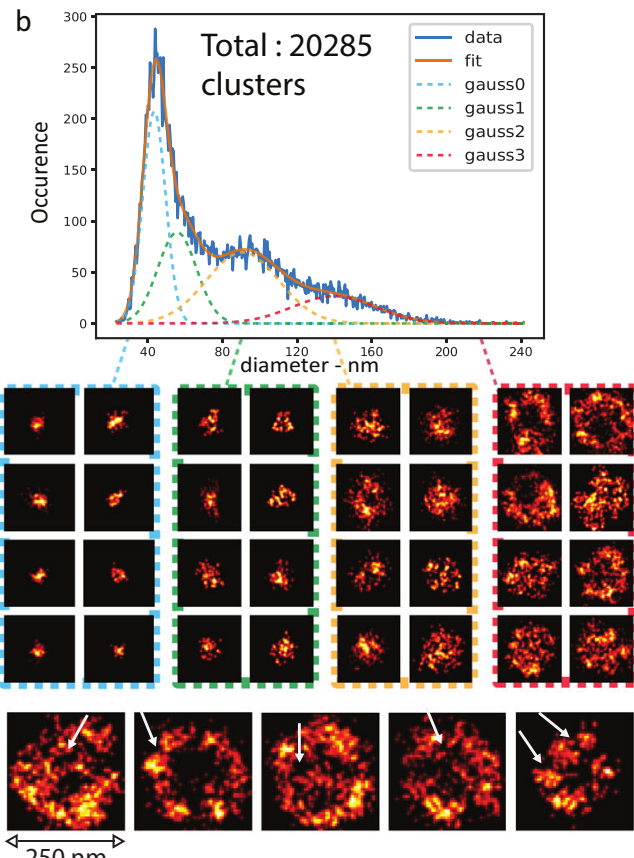

som
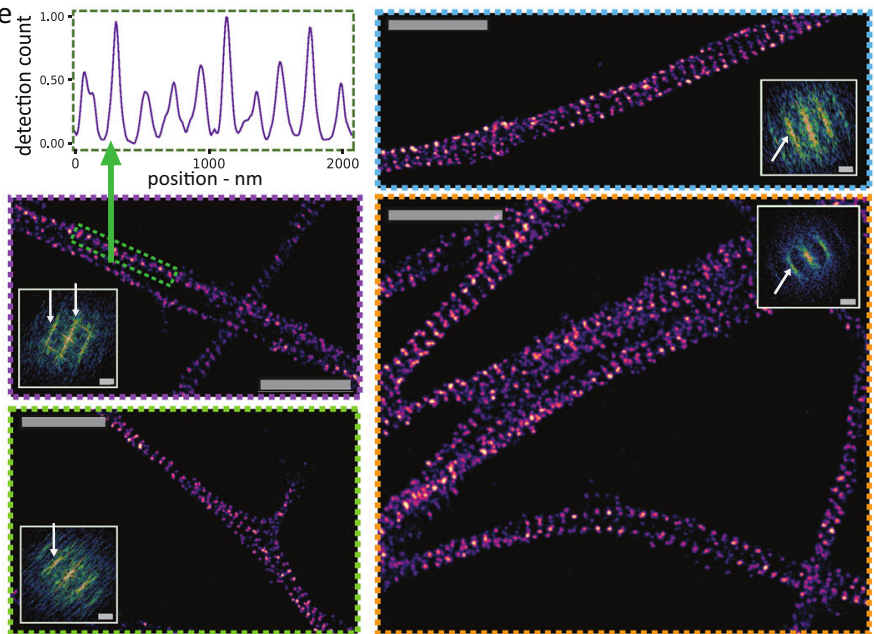

Fig. 5 ASTER applications for single-molecule localization microscopy. a-c ASTER STORM imaging and cluster analysis of COS-7 cells labeled for clathrin heavy-chain and an AF647-coupled secondary antibody. a Final $140 \mu \mathrm{m} \times 140 \mu \mathrm{m}$ image with scalebar $40 \mu \mathrm{m}$ (top left) and close-up views of the highlighted regions (colors encode cluster affiliation) with scalebars $1 \mu \mathrm{m}$. Pixel size is $10 \mathrm{~nm}$. b shows the distribution of the diameter of clathrin clusters and highlights four potential populations, that can be fitted with Gaussian functions. Below are $250 \mathrm{~nm} \times 250 \mathrm{~nm}$ images of individual clathrin related clusters, each group corresponding to a specific population. c shows $250 \mathrm{~nm} \times 250 \mathrm{~nm}$ images of large, hollow clathrin clusters likely corresponding to large clathrin-coated pits. Visible cavities are highlighted by arrows. d, e ASTER STORM imaging and structural analysis of neurons labeled for $\beta 2$-spectrin and AF647-coupled secondary antibody. d $200 \mu \mathrm{m} \times 200 \mu \mathrm{m}$ STORM image obtained with ASTER (30 nm pixel size). Scalebar $40 \mu \mathrm{m}$. The two-

dimensional Fourier transformation (inset) exhibits a circular frequency pattern corresponding to a $190 \mathrm{~nm}$ periodicity of the staining that is present along all axons. e Zoomed views of regions in $\mathbf{d}$ revealing the periodic cytoskeleton along single axons (pixel size is 10 nm). Upper left image shows the intensity profile along the highlighted green line, revealing again the $190 \mathrm{~nm}$ periodicity. Scalebars $2 \mu \mathrm{m}$. For $\mathbf{d}$, e, insets show the respective two-dimensional Fourier transformation and the known $190 \mathrm{~nm}$ periodicity of the axonal spectrin scaffold is highlighted by arrows. All Fourier images scalebars are $4 \mu m^{-1}$.

may be used in combination with improved detection schemes, such as multicolor imaging or strategies to encode axial information $^{50,51}$ (see Supplementary Fig. 15). It could be beneficial to setups that modify the excitation to enhance resolution by counting photons ${ }^{52-55}$ but ASTER implementation in such case would be challenging. The resulting uniformity may be used for demultiplexing or stoichiometry experiments ${ }^{56,57}$, as well as in buffer characterization and other fields such as photolitography ${ }^{58,59}$. Finally, ASTER has potential applications in nonuniform excitation schemes, such as using smaller beams to concentrate power ${ }^{60}$, exciting specific areas of a sample ${ }^{61}$ or creating a patterned irradiance on complex samples by using adaptive scanning strategies.

\section{Methods}

Optical setup. We used a Nikon Eclipse Ti inverted microscope with a Nikon Perfect Focus System. The excitation was performed with an ELERA laser $(638 \mathrm{~nm})$ from ERROL. $6215 \mathrm{H}$ galvanometers from Cambridge Technology were controlled with a RIGOL DG5252 waveform generator. To maintain telecentricity, all distances between lenses are equal to the sum of their respective focal lengths. Both excitation and detection went through the left camera port of the microscope to prevent undesired cropping. To this end, the dichroic is put in front of the side port and reflects the excitation beam (not shown in Fig. 1). Fluorescence was collected 
through an Olympus $\times 601.49 \mathrm{NA}$ oil immersion objective, a relay-system, and recorded on a $2048 \times 2048$ pixel sCMOS camera (Orca-Flash 4 v3, Hamamatsu) The optical pixel size was $\sim 108 \mathrm{~nm}$.

\section{Calibration sample preparation and imaging}

Beads. Beads are $3 \mu \mathrm{m}$ radius biotin-polystyrene microspheres (Kisker Biotech, PCB-3.0) on which we attached Alexa Fluor (AF) 647 functionalized with streptavidin (Life Technologies, S21374). We prepared a solution containing $500 \mu \mathrm{l}$ of water, $500 \mu \mathrm{l}$ of PBS, $35 \mu \mathrm{l}$ of microsphere solution, and $0.34 \mu \mathrm{l}$ of AF647. This solution was centrifuged $20 \mathrm{~min}$ at $12,100 \times g$. The liquid was then removed and replaced with $100 \mu \mathrm{l}$ of PBS, followed by $5 \mathrm{~min}$ vortexing to dissolve the deposit. In total, $50 \mu \mathrm{l}$ of the final solution was then pipetted on to a glass coverslip and left for $20 \mathrm{~min}$ so that beads would have time to deposit. Finally, we added $500 \mu \mathrm{l}$ of imaging dSTORM buffer (dSTORM smart kit, Abbelight). Images were taken at low laser power and integrated over $100 \mathrm{~ms}$, for an ASTER scan period of $50 \mathrm{~ms}$.

Nanorulers. Nanorulers (Gattaquant, PAINT-40R) consist in three aligned spots, separated by $40 \mathrm{~nm}$ and are labeled with ATTO655 fluorophores. To switch from ASTER to a Gaussian illumination, a constant offset was applied to galvanometers and a beam magnifier was placed between the laser and galvanometers. Parameters for imaging were chosen while optimizing the blinking with the wide Gaussian excitation $(\sigma=45 \mu \mathrm{m})$ : laser power of $200 \mathrm{~mW}$, fixed TIRF configuration, and an integration time of $100 \mathrm{~ms}$; parameters were maintained for each acquisition. During the acquisition on the $120 \mu \mathrm{m} \times 120 \mu \mathrm{m}$ FOV, the blinking of fluorophores was slow, so fluorophores generally appeared on subsequent frames and required larger integration times or post-processing to merge them. This became apparent in the loss in resolution.

\section{Fluorescence immunolabeling}

Neuronal culture. Rat hippocampal neurons in culture were prepared according to the Banker protocol ${ }^{62}$. Briefly, E18 Wistar rat embryo hippocampi (Janvier labs) were dissected, then cells were homogenized and plated in B27-containing Neurobasal medium on Poly-L-Lysine treated \#1.5H glass coverslips (Marienfeld, VWR) to a density of 4000 cells per $\mathrm{cm}^{2}$. The neurons were then co-cultured with glia cells-neuron coverslip upside down, separated from the glia on the bottom of the petri dish by wax beads. Mature neurons were fixed after 14 days in culture. All procedures followed the guidelines from European Animal Care and Use Committee (86/609/CEE) and were approved by Aix-Marseille university ethics committee (agreement D13-055-8).

Immunolabeling of neurons was performed according to recent optimized SMLM sample preparations ${ }^{47,63}$ : neurons were fixed using $4 \%$ paraformaldehyde (Delta Microscopie, \#15714) and 4\% (w/v) sucrose in PEM buffer (80 mM PIPES, $2 \mathrm{mM} \mathrm{MgCl}_{2}, 5 \mathrm{mM}$ EGTA, pH 6.8) for $20 \mathrm{~min}$ at RT. Cells were then rinsed with $0.1 \mathrm{M}$ phosphate buffer. Blocking and permeabilization were performed in ICC buffer $(0.2 \%(\mathrm{v} / \mathrm{v})$ gelatin, $0.1 \%$ Triton X-100 in phosphate buffer) for $2 \mathrm{~h}$ on a rocking table. Primary antibodies diluted in ICC were incubated overnight at $4{ }^{\circ} \mathrm{C}$, rinsed and incubated with the secondary antibodies diluted in ICC for $1 \mathrm{~h}$ at room temperature. After a final rinse with ICC and phosphate buffer, the samples were stored in phosphate buffer with $0.02 \%(\mathrm{w} / \mathrm{v})$ sodium azide before imaging. For immunolabeling, we used mouse anti $\beta 2$-spectrin (BD Sciences, \#612563, $2.5 \mu \mathrm{g} / \mathrm{ml}$ ) and donkey anti-mouse AF647 (Thermo Fisher, \#A31571, $6.67 \mu \mathrm{g} / \mathrm{ml}$ ).

Cell line culture. COS-7 cells were grown in DMEM with $10 \% \mathrm{FBS}, 1 \%$ L-glutamin, and $1 \%$ penicillin/streptomycin (Life Technologies) at $37^{\circ} \mathrm{C}$ and $5 \% \mathrm{CO}_{2}$ in a cell culture incubator. They were plated at medium confluence on cleaned, round $25 \mathrm{~mm}$ diameter high resolution 1.5" glass coverslips (Marienfield, VWR). After $24 \mathrm{~h}$, the cells were washed three times with PHEM solution (60 mM PIPES, $25 \mathrm{mM}$ HEPES, $5 \mathrm{mM}$ EGTA, and $2 \mathrm{mM} \mathrm{Mg}$ acetate adjusted to $\mathrm{pH} 6.9$ with $1 \mathrm{M}$ $\mathrm{KOH})$. For preparation of STORM microtubule imaging, we added an extraction solution ( $0.25 \%$ Triton, $0.025 \%$ Glutaraldehyde in PEM) for $30 \mathrm{~s}$ then a fixation solution $(0.5 \%$ glutaraldehyde, $0.5 \%$ Triton in PEM) for 12 min followed by a reduction solution $\left(\mathrm{NaBH}_{4}: 0.1 \%\right.$ in PBS $\left.1 \mathrm{X}\right)$ for $7 \mathrm{~min}$. For clathrin, we directly fixed with a 4\% PFA solution. Extraction and fixation solutions were pre-warmed at $37^{\circ} \mathrm{C}$. Cells were then washed three times in PBS before being blocked for 15 min in PBS $+1 \%$ BSA $+0.1 \%$ Triton. Labeling was performed in a similar solution with intermediary washing steps. $\alpha$-tubulin (Sigma Aldrich, T6199) and clathrin heavy-chain (Abcam, ab2731) primary antibodies were conjugated with Rb-AF647 (Life Technologies, A21237). Cells were finally postfixed for $16 \mathrm{~min}$ in $3.7 \%$ formaldehyde and reduced for $10 \mathrm{~min}$ with $\mathrm{NH}_{4} \mathrm{Cl}(3 \mathrm{mg} / \mathrm{ml})$.

\section{Biological sample imaging}

Widefield fluorescence imaging. TIRF imaging on neuronal sample was done at $200 \mathrm{~ms}$ integration times and a low $30 \mathrm{~mW}$ laser power. Samples consisted in $\beta 2$ spectrin labeled with AF647. Output angle was adjusted with a translation stage ${ }^{64}$ until penetration depth was roughly around $200 \mathrm{~nm}$.

STORM imaging. STORM imaging on COS-7 cells (microtubules and clathrin) and neurons ( $(32$-spectrin) was performed at $50 \mathrm{~ms}$ exposure time using a HiLo illumination configuration. A STORM buffer (Abbelight Smart kit) was used to induce most of the molecules in a dark state. The sample was lit with laser powers of $\sim 250 \mathrm{~mW}$ in the objective BFP and a scanned Gaussian beam of $\sigma=17 \mu \mathrm{m}$. Except for clathrin, all data acquisition was excited with an ASTER excitation scanning ten lines in $25 \mathrm{~ms}$ and a gap of $1.4 \sigma$. For STORM on clathrin, the labeling was dense and blinking was slightly optimized by reducing the excited FOV to $140 \mu \mathrm{m} \times$ $140 \mu \mathrm{m}$, scanning eight lines in $25 \mathrm{~ms}$ with a gap of $1.2 \sigma$. The acquisitions were performed and analyzed using the Nemo software (Abbelight). Localization consisted in a wavelet segmentation after median background removal, followed by Gaussian fits of individual point spread functions. Sample was drift-corrected using a classical redundant cross-correlation algorithm.

Image acquisition, processing, and analysis. Neo-Live (Abbelight) was used for image acquisition. Single-molecule analysis was performed either with a homemade Python 3.7 code or with Neo-Analysis (Abbelight).

Data processing such as analysis and measurement of beads radius from Fig. 2, nanoruler from Fig. 3, and clathrin from Fig. 5 was performed in Python, whose code is available online.

Beads. Beads (microspheres) were detected on the TIRF image: we first applied a Laplace filter from scipy library, followed by low-pass filtering in Fourier space to diminish noise. Use of an intensity threshold then proved sufficient to efficiently detect individual beads. Peaks positions were measured via local extrema algorithms.

Nanorulers. Nanorulers analysis focused on resulting $X, Y$ coordinates. A preliminary DBscan clustering was used to localize and filter out lonesome localizations. Then a more precise DBscan was used to distinguish individual groups of three-spots and associate a number to each of them. DBscan typically consider core points, which are point with at least mpts neighbor in a surrounding epsilon radius, then iteratively add adjacent points. Parameters of this secondary scan were: epsilon $=50 \mathrm{~nm}$ and minimum number of points mpts $=10$. With these parameters, adjacent spots belonging to a similar nanoruler array were grouped together, while unwanted associations of adjacent nanorulers were minimized. For each group, a GMM clustering was used to estimate parameters from three Gaussian distribution. GMM also estimates the mean and standard deviation of each spot, which allowed for size estimation and localization precision measurements. Nanorulers with too few points or extraordinary distance estimations were thrown away.

Clathrin clusters. Clathrin analysis was primarily performed via a DBscan clustering, with an epsilon parameter of $35 \mathrm{~nm}$ and a minimum number of points of 25. This clustering method localized each individual cluster of close points. For each of these cluster we calculated several parameters, such as the mean position and the effective diameter, Feret's diameter ${ }^{65}$, the hollowness, the angle of orientation, and eccentricity. Effective diameter and mean position were calculated by minimizing radial dispersion among points. Hollowness consisted in the ratio between the mean radius value, divided by the standard deviation of radius. Among all parameters, the diameter and the hollowness proved to be the most relevant in term of describing cluster distributions.

Neurons. Fourier transform was performed on $2 \mathrm{D}$ histogram images via the $\mathrm{ftt} 2$ function from the numpy.fft library of Python.

Reproducibility. Experiments with Nile Blue have been repeated five times, and yield similar results (Fig. 1b). When investigating the presence of vignetting, full field images of Nile Blue samples have been performed three times (Supplementary Fig. 3).

The effect of optical sectioning on beads was observed multiple times $(n>5)$ on three different samples (Supplementary Figs. 5 and 6). Optical imaging from EPI to TIRF, of neurons or other biological components, is extremely consistent: it has been performed multiple times $(n>20)$ on different setups (Fig. 2). The ability of our implementation to perform TIRF images with a scanning period of $5 \mathrm{~ms}$ has been repeated four times on the same sample, and should extend to any setup using similar scanning devices (Supplementary Fig. 7). Investigation of the difference between classical TIRF, ASTER TIRF, and spinning TIRF images has been realized three times (Supplementary Fig. 8).

Concerning SMLM Experiments, nanoruler PAINT experiments have been repeated three times for similar illumination configuration as presented in the manuscript, and yielded similar results (Fig. 3).

In total, $200 \mu \mathrm{m} \times 200 \mu \mathrm{m}$ direct imaging in STORM has been repeated at least eight times on different samples (Fig. 4). Cluster analyses of clathrin has been performed on two samples, only one of which is shown in the manuscript (Fig. $5 \mathrm{a}-\mathrm{c}$ ). Wide FOV imaging of neurons has been repeated five times (Fig. 5d and Supplementary Fig. 14).

For comparison with ASTER, Gaussian illumination in STORM has been performed twice (Supplementary Fig. 10). We have performed two stitching experiments, only one of which is shown in the supplementary (Supplementary Fig. 12). Concerning the ability to perform fast STORM imaging, it has been 
repeated consistently on two different setups and numerous samples $(n>10)$ (Supplementary Fig. 13).

Investigation of the effect of the scanning speed on STORM blinking kinetics has been performed once (Supplementary Fig. 11). 3D Images of microtubules on a $200 \mu \mathrm{m} \times 200 \mu \mathrm{m}^{2}$ FOV has been performed thrice (Supplementary Fig. 15).

Reporting summary. Further information on research design is available in the Nature Research Reporting Summary linked to this article.

\section{Data availability}

Data that support the findings of this study have been deposited on Zenodo (DOI: 10.5281/zenodo.4625796), with the exception of SMLM large raw data files (>20Go), which are available from the corresponding author on reasonable request.

\section{Code availability}

Code is available online on Github at the following link: https://github.com/AdrienMau/ ASTER_code and Zenodo (https://doi.org/10.5281/zenodo.4625796).

Received: 9 May 2020; Accepted: 26 April 2021;

Published online: 24 May 2021

\section{References}

1. Tokunaga, M., Imamoto, N. \& Sakata-Sogawa, K. Highly inclined thin illumination enables clear single-molecule imaging in cells. Nat. Methods 5, 159-161 (2008).

2. Nakata, T. \& Hirokawa, N. Microtubules provide directional cues for polarized axonal transport through interaction with kinesin motor head. J. Cell Biol. 162 , 1045-1055 (2003).

3. Guo, Y. et al. Visualizing intracellular organelle and cytoskeletal interactions at nanoscale resolution on millisecond timescales. Cell 175, 1430-1442 (2018).

4. Stout, A. L. \& Axelrod, D. Evanescent field excitation of fluorescence by epiillumination microscopy. Appl. Opt. 28, 5237-5242 (1989).

5. Axelrod, D. Cell-substrate contacts illuminated by total internal reflection fluorescence. J. Cell Biol. 89, 141-145 (1981).

6. Stock, K. et al. Variable-angle total internal reflection fluorescence microscopy (VA-TIRFM): realization and application of a compact illumination device. J. Microsc. 211, 19-29 (2003).

7. Konopka, C. A. \& Bednarek, S. Y. Variable-angle epifluorescence microscopy: a new way to look at protein dynamics in the plant cell cortex. Plant J. 53, 186-196 (2008).

8. van't Hoff, M., de Sars, V. \& Oheim, M. A programmable light engine for quantitative single molecule TIRF and HILO imaging. Opt. Express 16, 18495-18504 (2008).

9. Mattheyses, A. L., Shaw, K. \& Axelrod, D. Effective elimination of laser interference fringing in fluorescence microscopy by spinning azimuthal incidence angle. Microsc. Res. Tech. 69, 642-647 (2006)

10. Fiolka, R., Belyaev, Y., Ewers, H. \& Stemmer, A. Even illumination in total internal reflection fluorescence microscopy using laser light. Microsc. Res. Tech. 71, 45-50 (2008).

11. Boulanger, J. et al. Fast high-resolution 3D total internal reflection fluorescence microscopy by incidence angle scanning and azimuthal averaging. Proc. Natl Acad. Sci. USA 111, 17164-17169 (2014).

12. Schreiber, B., Elsayad, K. \& Heinze, K. G. Axicon-based Bessel beams for flatfield illumination in total internal reflection fluorescence microscopy. Opt. Lett. 42, 3880-3883 (2017).

13. Almada, P., Culley, S. \& Henriques, R. PALM and STORM: into large fields and high-throughput microscopy with sCMOS detectors. Methods $\mathbf{8 8}$ 109-121 (2015).

14. Betzig, E. et al. Imaging intracellular fluorescent proteins at nanometer resolution. Science 313, 1642-1645 (2006).

15. Hess, S. T., Girirajan, T. P. K. \& Mason, M. D. Ultra-high resolution imaging by fluorescence photoactivation localization microscopy. Biophys. J. 91, 4258-4272 (2006).

16. Sharonov, A. \& Hochstrasser, R. M. Wide-field subdiffraction imaging by accumulated binding of diffusing probes. Proc. Natl Acad. Sci. USA 103, 18911-18916 (2006).

17. Rust, M. J., Bates, M. \& Zhuang, X. Sub-diffraction-limit imaging by stochastic optical reconstruction microscopy (STORM). Nat. Methods 3, 793-796 (2006).

18. Heilemann, M. et al. Subdiffraction-resolution fluorescence imaging with conventional fluorescent probes. Angew. Chem. Int. Ed. 47, 6172-6176 (2008).
19. Heilemann, M., van de Linde, S., Mukherjee, A. \& Sauer, M. Super-resolution imaging with small organic fluorophores. Angew. Chem. Int. Ed. 48, 6903-6908 (2009)

20. Ramachandran, S., Cohen, D. A., Quist, A. P. \& Lal, R. High performance, LED powered, waveguide based total internal reflection microscopy. Sci. Rep. 3, 2133 (2013).

21. Diekmann, R. et al. Chip-based wide field-of-view nanoscopy. Nat. Photonics 11, 322-328 (2017).

22. Archetti, A. et al. Waveguide-PAINT offers an open platform for large fieldof-view super-resolution imaging. Nat. Commun. 10, 1267 (2019).

23. Douglass, K. M., Sieben, C., Archetti, A., Lambert, A. \& Manley, S. Superresolution imaging of multiple cells by optimized flat-field epi-illumination. Nat. Photonics 10, 705-708 (2016).

24. Khaw, I. et al. Flat-field illumination for quantitative fluorescence imaging. Opt. Express 26, 15276-15288 (2018).

25. Rowlands, C. J., Ströhl, F., Ramirez, P. P. V., Scherer, K. M. \& Kaminski, C. F. Flat-field super-resolution localization microscopy with a low-cost refractive beam-shaping element. Sci. Rep. 8, 5630 (2018).

26. Stehr, F., Stein, J., Schueder, F., Schwille, P. \& Jungmann, R. Flat-top TIRF illumination boosts DNA-PAINT imaging and quantification. Nat. Commun. 10, 1268 (2019).

27. Zhao, Z., Xin, B., Li, L. \& Huang, Z.-L. High-power homogeneous illumination for super-resolution localization microscopy with large field-ofview. Opt. Express 25, 13382-13395 (2017).

28. Deschamps, J., Rowald, A. \& Ries, J. Efficient homogeneous illumination and optical sectioning for quantitative single-molecule localization microscopy. Opt. Express 24, 28080-28090 (2016).

29. Kwakwa, K. et al. easySTORM: a robust, lower-cost approach to localisation and TIRF microscopy. J. Biophotonics 9, 948-957 (2016).

30. Chen, S.-Y., Bestvater, F., Schaufler, W., Heintzmann, R. \& Cremer, C. Patterned illumination single molecule localization microscopy (piSMLM): user defined blinking regions of interest. Opt. Express 26, 30009-30020 (2018)

31. Kurvits, J. A., Jiang, M. \& Zia, R. Comparative analysis of imaging configurations and objectives for Fourier microscopy. J. Opt. Soc. Am. A 32, 2082-2092 (2015)

32. Mattheyses, A. L. \& Axelrod, D. Direct measurement of the evanescent field profile produced by objective-based total internal reflection fluorescence. $J$. Biomed. Opt. 11, 014006 (2006).

33. Cabriel, C., Bourg, N., Dupuis, G. \& Lévêque-Fort, S. Aberration-accounting calibration for 3D single-molecule localization microscopy. Opt. Lett. 43, 174-177 (2018).

34. Ester, M., Kriegel, H.-P., Sander, J. \& Xu, X. A density-based algorithm for discovering clusters in large spatial databases with noise. In Proceedings of the Second International Conference on Knowledge Discovery and Data Mining 226-231 (AAAI Press, 1996).

35. Tam, J., Cordier, G. A., Borbely, J. S., Sandoval Álvarez, Á. \& Lakadamyali, M. Cross-talk-free multi-color STORM imaging using a single fluorophore. PLoS ONE 9, e101772 (2014).

36. Xu, J., Ma, H. \& Liu, Y. Stochastic optical reconstruction microscopy (STORM). Curr. Protoc. Cytom. 81, 12.46.6-12.46.27 (2017).

37. Lin, Y. et al. Quantifying and optimizing single-molecule switching nanoscopy at high speeds. PLoS ONE 10, e0128135 (2015).

38. Banterle, N., Bui, K. H., Lemke, E. A. \& Beck, M. Fourier ring correlation as a resolution criterion for super-resolution microscopy. J. Struct. Biol. 183, 363-367 (2013).

39. Bates, M., Blosser, T. R. \& Zhuang, X. Short-range spectroscopic ruler based on a single-molecule optical switch. Phys. Rev. Lett. 94, 108101 (2005).

40. Diekmann, R. et al. Optimizing imaging speed and excitation intensity for single-molecule localization microscopy. Nat. Methods 17, 909-912 (2020)

41. Heuser, J. Three-dimensional visualization of coated vesicle formation in fibroblasts. J. Cell Biol. 84, 560-583 (1980).

42. Lampe, M., Vassilopoulos, S. \& Merrifield, C. Clathrin coated pits, plaques and adhesion. J. Struct. Biol. 196, 48-56 (2016).

43. Leterrier, C. et al. Nanoscale architecture of the axon initial segment reveals an organized and robust scaffold. Cell Rep. 13, 2781-2793 (2015).

44. Ganguly, A. et al. A dynamic formin-dependent deep F-actin network in axons. J. Cell Biol. 210, 401-417 (2015).

45. Xu, K., Zhong, G. \& Zhuang, X. Actin, spectrin, and associated proteins form a periodic cytoskeletal structure in axons. Science 339, 452-456 (2013).

46. Leterrier, C., Dubey, P. \& Roy, S. The nano-architecture of the axonal cytoskeleton. Nat. Rev. Neurosci. 18, 713-726 (2017).

47. Vassilopoulos, S., Gibaud, S., Jimenez, A., Caillol, G. \& Leterrier, C. Ultrastructure of the axonal periodic scaffold reveals a braid-like organization of actin rings. Nat. Commun. 10, 5803 (2019).

48. Potsaid, B., Bellouard, Y. \& Wen, J. T. (ASOM): a multidisciplinary optical microscope design for large field of view and high. Opt. Express 13, 6504-6518 (2005). 
49. Potsaid, B. et al. Living organism imaging with the adaptive scanning optical microscope (ASOM). Proc. SPIE 6441, Imaging, Manipulation, and Analysis of Biomolecules, Cells, and Tissues 6441, 64411D (SPIE, 2007).

50. Huang, B., Wang, W., Bates, M. \& Zhuang, X. Three-dimensional superresolution imaging by stochastic optical reconstruction microscopy. Science 319, 810-813 (2008).

51. Cabriel, C. et al. Combining 3D single molecule localization strategies for reproducible bioimaging. Nat. Commun. 10, 1980 (2019).

52. Cnossen, J. et al. Localization microscopy at doubled precision with patterned illumination. Nat. Methods 17, 59-63 (2020).

53. Reymond, L. et al. SIMPLE: structured illumination based point localization estimator with enhanced precision. Opt. Express 27, 24578-24590 (2019).

54. Eilers, Y., Ta, H., Gwosch, K. C., Balzarotti, F. \& Hell, S. W. MINFLUX monitors rapid molecular jumps with superior spatiotemporal resolution. Proc. Natl Acad. Sci. USA 115, 6117-6122 (2018).

55. Jouchet, P. et al. Nanometric axial localization of single fluorescent molecules with modulated excitation. Nat. Photonics 15, 297-304 (2021).

56. Bossi, M. et al. Multicolor far-field fluorescence nanoscopy through isolated detection of distinct molecular species. Nano Lett. 8, 2463-2468 (2008).

57. Fricke, F., Beaudouin, J., Eils, R. \& Heilemann, M. One, two or three? Probing the stoichiometry of membrane proteins by single-molecule localization microscopy. Sci. Rep. 5, 14072 (2015).

58. Hung, Y.-J., Chang, H.-J., Chang, P.-C., Lin, J.-J. \& Kao, T.-C. Employing refractive beam shaping in a Lloyd's interference lithography system for uniform periodic nanostructure formation. J. Vac. Sci. Technol. B 35, 030601 (2017).

59. Weber, D. et al. Use of beam-shaping optics for wafer-scaled nanopatterning in laser interference lithography. Appl. Phys. A 125, 307 (2019).

60. Ishikawa-Ankerhold, H., Ankerhold, R. \& Drummen, G. Fluorescence Recovery After Photobleaching (FRAP). In eLS (ed. John Wiley \& Sons Ltd, 2014).

61. Dreier, J. et al. Smart scanning for low-illumination and fast RESOLFT nanoscopy in vivo. Nat. Commun. 10, 556 (2019).

62. Kaech, S. \& Banker, G. Culturing hippocampal neurons. Nat. Protoc. 1, 2406-2415 (2006).

63. Jimenez, A., Friedl, K. \& Leterrier, C. About samples, giving examples: optimized single molecule localization microscopy. Methods 174, 100-114 (2020).

64. Bourg, N. et al. Direct optical nanoscopy with axially localized detection. Nat. Photonics 9, 587-593 (2015).

65. Feret, L. La grosseur des grains des matières pulvérulentes (1930).

\section{Acknowledgements}

We acknowledge people who provided valuable advice and support in the course of this study, particularly P. Jouchet and C. Cabriel, as well as the CPBM/ISMO-UMR8214 for access to cell culture facility. We thank S. Vassilopoulos for discussions about clathrin organization in cells. M. Bardou and F. Boroni-Rueda helped with cell culture and labeling. C. Hubert and E. Fort provided the lasers and galvanometer scanners.

\section{Author contributions}

A.M., N.B., and S.L.-F. conceived the project. All authors contributed to the writing and editing of the manuscript. K.F. and C.L. provided neuronal samples. Experimental acquisitions, analyses, and illustrations were performed by A.M.

\section{Competing interests}

N.B. and S.L.F. are shareholders in Abbelight. Other authors declare no competing interests.

\section{Additional information}

Supplementary information The online version contains supplementary material available at https://doi.org/10.1038/s41467-021-23405-4.

Correspondence and requests for materials should be addressed to S.L.-F.

Peer review information Nature Communications thanks Alistair Curd and Zhen-L Huang for their contribution to the peer review of this work. Peer reviewer reports are available.

Reprints and permission information is available at http://www.nature.com/reprints

Publisher's note Springer Nature remains neutral with regard to jurisdictional claims in published maps and institutional affiliations.

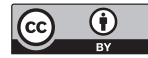

Open Access This article is licensed under a Creative Commons Attribution 4.0 International License, which permits use, sharing, adaptation, distribution and reproduction in any medium or format, as long as you give appropriate credit to the original author(s) and the source, provide a link to the Creative Commons license, and indicate if changes were made. The images or other third party material in this article are included in the article's Creative Commons license, unless indicated otherwise in a credit line to the material. If material is not included in the article's Creative Commons license and your intended use is not permitted by statutory regulation or exceeds the permitted use, you will need to obtain permission directly from the copyright holder. To view a copy of this license, visit http://creativecommons.org/ licenses/by/4.0/.

(C) The Author(s) 2021 